\title{
Array Enhancement of Stochastic Synchronization and Signal-to-Noise Ratio Gain in Nonlinear Regime of Signal Transmission
}

\author{
I. A. Khovanov* \\ Department of Physics, Lancaster University, Lancaster LA1 4YB, United Kingdom
}

\begin{abstract}
The nonlinear transformation of an external noisy signal by an array of non-interacting elements with internal noise is considered. The array simulates a neuronal system that processes spike trains. It is shown that increasing the number of array elements entails significant extending of the stochastic synchronization region and improvement of the signal-to-noise ratio (SNR). The effects are demonstrated for arrays of triggers, overdamped bistable oscillators and Fitzhugh-Nagumo systems. The interrelation between SNR improvement and the efficiency of information processing is discussed.
\end{abstract}

PACS numbers: 05.40.-a,05.45.Xt,05.50.+q,84.35.+i

Keywords: stochastic processes; synchronization; nonlinear dynamical systems; noise; neuron

\section{INTRODUCTION}

The interplay between noise and the nonlinearity of a system can produce nontrivial effects that lead to enhancement of the system response. A remarkable example of this is signal processing by a threshold element, when any sub-threshold signals cannot be detected without an additional noise component, and by tuning noise intensity one is able to optimize the detection. This example is a manifestation of a more general phenomenon called stochastic resonance (SR) $[1,2]$. Initially, the essential ingredients providing SR effect have included a bistable system, a periodic signal and white noise $[3,4]$, but later they were significantly extended $[1,2]$, and it has been shown that for a wide range of situations, noise is able to optimize the system response.

Unflagging interest in SR can be explained by the variety of possible combinations of system nonlinearity, signal and noise properties. Another important factor is the relevance of SR to biological processes, especially to neuroscience [5], where fluctuations persist from the molecular level (e.g. ion channels) to networks. This factor induces the study of information transformation via complex networks of elements with external and internal noise sources [5]. It has been shown that a network (or an array of elements) enhances significantly the system response in comparison with a single element [6-9]. The conclusion is valid for a variety of array configurations with or without coupling of elements, with different types of local and global couplings. In the neuroscience context, a parallel array of nonlinear elements with a summing center attracts a lot of attention [10-14]. It has been shown that internal noise in such a parallel array leads to the optimization of information transmission in a wide range of noise intensity. This optimization can be explained by noise-induced linearization of the nonlinear responses of individual elements [14, 15] and,

*Electronic address: i.khovanov@warwick.ac.uk consequently, the array response can be described in the framework of linear response theory [15].

In this contribution we consider a nonlinear regime of a parallel array with summing and nonlinear end elements. The array encodes an input signal into a sequence of switching events (spikes) [16], and the informational component of the input signal has the same structure as the output of the array. The nonlinear regime in the system is realized when the amplitude of the signal is large enough. Within the nonlinear regime, many different effects, for example, the generation of higher harmonics [17], can be observed. We concentrate our attention on stochastic synchronization [19] and signal-to-noise-ratio (SNR) improvement [20, 21].

The effect of stochastic synchronization is an important one from the signal processing point of view. Synchronization occurs when noise-induced switchings between system's states follow the phase of the external signal, and it is observed in the range of noise intensity $[18,19,22-25]$. In [26], it has been shown that switching synchronization can be realized for a random spike train, i.e noise induces switching for each spike. An experimental study of stochastic synchronization in a biological system has been done in [27]. This phenomenon is important for understanding signal processing in the neuronal system, since it specifies the conditions when noise induces a regime of complete (optimal) information transmission by spikes, and it is observed simultaneously over a wide range of noise amplitude.

In [18] an extensive analysis of stochastic synchronization in an array of threshold elements in the limit of a weak signal has been presented. The transformation of the signal with a finite input SNR by an array with elements perturbed by independent noise sources was analyzed using linear response theory. The finite input SNR means that the external signal consists of informational and noise components, whereas internal noise sources do not relate to the external noise. It is shown [18] that in the case of infinite input SNR (informational component only), synchronization can be observed for an arbitrary intensity of internal noise by increasing the number of array elements. In turn, the finite input SNR leads to 
a limitation on the maximal intensity of internal noise, and starting with a certain value of input SNR (intensity of the noise component) the synchronization does not occur. The main result of work [18] consists of the following. A large number of uncoupled elements perturbed by independent noise sources tends to "remove" the nonlinearity of each element, and the independence of noise sources "removes" output noise during summing. As a result, with increasing the number of elements, the array's response tends to be linear and noise-free, i.e. it repeats the shape of the input signal. Consequently, synchronization between input and output signals is observed. In the present paper the regime is fundamentally nonlinear, so for a large amplitude of input signal the described linearization does not occur, but the synchronization can still take place since a larger signal modulates strongly noise-induced switchings, for example, reaching the threshold of the excitable element is possible for a specific range of signal phase only (see [19, 24, 25, 28] for details).

SNR improving (gain) was observed experimentally in a number of systems $[20,21,29]$ and corresponding theoretical support of the experimental results has also been provided later[30]. The gain means that the output value of SNR exceeds the input value [30]. In [21, 31], SNR gain in connection with switching statistics has been discussed. Recently, Cubero et al [32] have numerically shown that an array of coupled nonlinear elements is able to increase significantly SNR gain in comparison with a linear array ${ }^{1}$. Note that the presence of SNR gain is usually interpreted as an enhancement of signal transmission, so we further discuss the interrelation between SNR gain and the efficiency of signal processing.

The configuration of the considered array is shown in Fig. 1. The main difference from previously discussed configurations $[10-14,18]$ is the presence of the last (end) nonlinear element, $T_{S}$, following after a summing center $\sum$. The nonlinear element $T_{S}$ is characterized by the same nonlinearity as the base element, $T_{i}$. This modification leads to the configuration used for neuronal networks in the visual system [5] and corresponds to the cascade model of the neuron [33]. In this case input information is encoded by the output spike train (a sequence of switchings), but not by the shape of the input signal [1013]. In previous investigations [10-14, 18], arrays of both bistable and excitable elements have been considered. In fact, both types of elements are used as a base element of neuronal networks $[5,34]$. Therefore, we consider both of them in order to analyze the role of the base element's dynamics. In Sec. II we investigate arrays of two bistable units: Schmitt triggers and bistable overdamped oscillators. An array of excitable elements is discussed in Sec. III. The conclusions drawn are summarized in Sec. IV.

1 We have to note that some previous reported results of SNR gain in arrays raised some questions (see comment [31] for details).
Technical details of numerical simulations are presented in the Appendix.

\section{BISTABLE ARRAY}

Let us, first, consider the Schmitt trigger as a base element. The trigger is the simplest non-dynamical bistable system with a pure two-state dynamics defined by its hysteresis loop. In the case of a symmetric loop, the trigger's dynamics is described by the following equation:

$$
\begin{gathered}
U(t)=U_{0} \operatorname{sgn}(\Delta U-F(t)), \\
F(t)=F_{i}(t)+\xi_{c}(t)+\xi_{i}(t) ;
\end{gathered}
$$

here $F(t)$ is an input signal; "sgn" is the sign function; $\Delta U=|\Delta U| \operatorname{sgn}[U(t)]$ are the trigger's thresholds; the output value $U(t)$ is equal to either positive or negative $U_{0}$, i.e. $U(t)= \pm U_{0}$, and it is controlled by the signal $F(t)$, i.e. if $F(t)>|\Delta U|$, the trigger is in its upper state $+U_{0}$, and if $F(t)<-|\Delta U|$, then it is in the state $-U_{0}$.

The input signal $F(t)$ consists of three components. The informational component $F_{i}(t)$ is a periodic sequence of rectangular pulses with frequency $f=1 / T$ (or angular frequency $\Omega=2 \pi / T)$ and amplitude $A$ : $F_{i}(t)=$ $(-1)^{m(t)} A$, where $m(t)=\lfloor 2 t / T\rfloor,\lfloor x\rfloor=\max \{n \in \mathbb{Z} \mid n \leq$ $x\}$ is the floor function of $x$. The amplitude $A$ is comparable with, but less than, the threshold value, i.e. the informational component is a sub-threshold one and cannot be detected without some additional components. The second component is common noise $\xi_{c}(t)$, superimposed on pulses $F_{i}(t)$. The third component is internal additive noise $\xi_{i}(t)$ acting independently in each element. Note that a 3-component input was used in [18], whereas in most cases $[10-14,32]$, the common noise, $\xi_{c}(t)$, is not considered. Both noises are modeled as twice low-pass filtered Gaussian white noise $\eta(t)$ (see [35] for details) with identical cut-off frequencies, $1 / \tau=100$, for both filters:

$$
\ddot{\xi}+\frac{2}{\tau} \dot{\xi}+\frac{1}{\tau^{2}} \xi=\frac{\sqrt{2 D}}{\tau} \eta(t) .
$$

The intensities of the white noise sources are $D_{c}$ for common noise and $D$ for the internal noise sources. If $D_{c} \neq 0$, then a signal with a finite SNR acts on each element.

The sum of trigger outputs $S(t)=\sum_{i}^{N} U^{i}(t)$ acts on trigger $T_{s}$, which in turn produces the output of the considered array $U_{\text {out }}$. Our aim is to consider the array performance as a function of the intensity $D$ of internal noise. To this aim, two measures are discussed: The mean switching rate (MSR), $\langle f\rangle$, (or angular frequency $\langle\omega\rangle=2 \pi\langle f\rangle)$ and SNR, $R$. The MSR is calculated as the mean number of switchings during the calculation time. Alternatively, one can use a signal processing measure like the receiver operating characteristic $[8,36]$ or the total error, as applied in neuroscience [37] (see below). Since we consider the regime of synchronization in the system with two symmetric thresholds, the use of MSR 


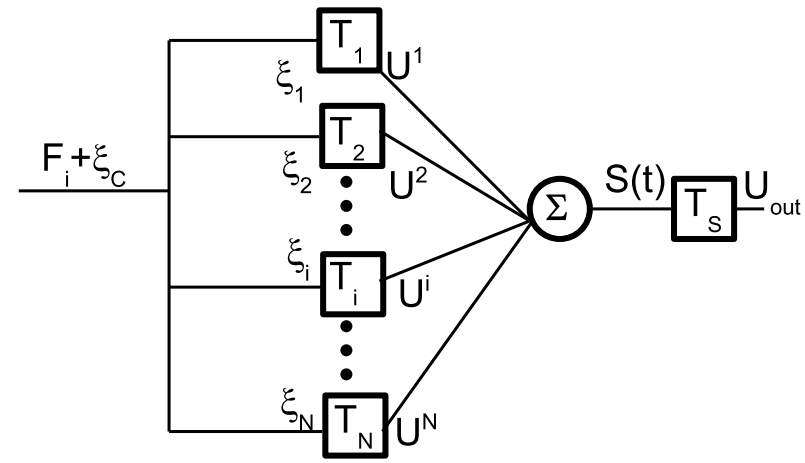

FIG. 1: The configuration of the considered array of elements.

is sufficient to characterize a signal transmission via the bistable array.

The SNR is defined by analogy with the conventional definition, used for a harmonic signal in a noisy linear system, as the common logarithm of the ratio between the weight of the $\delta$-peak, $S_{i}$, in the power spectrum at the pulse repetition frequency $\Omega$, to the intensity of the noisy background, $S_{n}$, at the same frequency [30]:

$$
R=10 \log _{10} \frac{S_{i}(\Omega)}{S_{n}(\Omega)}
$$

and it is measured units of decibels $(\mathrm{dB})$. The definition is based on the fact $[30,31,38,39]$ that the output of a periodically forced system is a superposition of periodic and noisy components.

Let us consider the dynamics of the array in the absence of common noise $\xi_{c}(t)$, i.e. $D_{c}=0$. The population dynamics of the single element $n_{ \pm}^{i}(t)$ are defined by the probability that $i$-element is in one of the states $+U_{0}$ or $-U_{0}$. The rate equations for populations $n_{ \pm}(t)$ have the forms $[40,41]$

$$
\begin{gathered}
\dot{n}_{+}=-r_{-} n_{+}+r_{+} n_{-} \\
\dot{n}_{-}=-r_{+} n_{-}+r_{-} n_{+},
\end{gathered}
$$

where $r_{ \pm}(t)$ are the threshold crossing rates. For the trigger, the rates are [35]:

$$
r_{ \pm}(t)=\frac{1}{2 \pi \tau} \exp \left(-\frac{\left[ \pm|\Delta U|-F_{i}(t)\right]^{2} \tau}{D}\right) .
$$

Note that using expression (5) has provided good correspondence between theoretical and experimental results (see [25] for details). In Ref. [30], it has been demonstrated that in the case of periodic pulses, the long-time solution of (4) has the form:

$$
n_{+}(t)=-\frac{1}{2}+\frac{F_{i}(t) \Delta r}{2 A}\left[1+\Delta r \frac{e^{-\gamma(t-m(t) T / 2)}}{1+e^{-\gamma T / 2}}\right],
$$

where $\gamma=r_{+}(0)+r_{-}(0), \Delta r=\left[r_{-}(0)-r_{+}(0)\right] / \gamma$. Expression (6) specifies a periodic function, i.e. $n_{+}(t)=$
$n_{+}(t+T)$ for any $t$. In the regime when the switching rate is comparable with the frequency $f$, the last term in the brackets is practically constant, and the time dependence of the population coincides with the input signal $F_{i}(t)$, i.e. it is a rectangular shape function with some maximal $n_{+}^{\max }$ and minimal values $n_{+}^{\text {min }}$ (note that $n_{+}^{\max }=1-n_{-}^{\text {min }}$ and $n_{+}^{\min }=1-n_{-}^{\max }$ ). In the synchronization regime, $n_{ \pm}^{\max }$ is close to the unit value, whereas $n_{ \pm}^{\min }$ is close to zero: $n_{ \pm}^{\max }>1-\epsilon, n_{ \pm}^{\min }<\epsilon$, $\max _{t} r_{ \pm}(t)>f, \epsilon \ll 1$. In other words, the difference $\Delta n^{m}=n_{ \pm}^{\max }-n_{ \pm}^{\min }>1-2 \epsilon$ is close to the unit value.

The input of the end element $T_{s}$ is the sum of outputs $S(t)=\sum_{i}^{N} U^{i}(t)$, and $S(t)$ is a random discrete-value variable distributed accordingly to the time-dependent Bernoulli law:

$$
P[S(t)]=C_{j}^{N} n_{+}^{j}(t)\left[1-n_{+}(t)\right]^{N-j},
$$

where $j=0.5(S-N)$ and $j=0,1,2 \ldots N, C_{j}^{N}=$ $N ! /(j !(N-j) !)$ are binomial coefficients, and $n_{+}(t)$ is the time dependent population of $i$-element. The discretevalue signal $S(t)$ can be approximated by a continuous signal with a normal distribution [42], which is defined by a time dependent mean value $\mu(t)=N\left[2 n_{+}(t)-1\right]$ and a variance $\sigma^{2}(t)=2 N n_{+}(t)\left[1-n_{+}(t)\right]$. Dynamics of the mean value $\mu(t)$ is determined by the population dynamics $n_{+}(t)$ and consequently it is a periodic function of time and repeats the shape of $n_{+}(t)$. So, in the synchronization regime $\mu(t)$ is a rectangular shape function with some maximal and minimal values $\pm \mu^{m}$ and a very narrow switching region in the case $\max _{t} r_{ \pm}(t)>f$. If $\mu^{m}$ is larger than the threshold $|\Delta U|$ and it is larger than $4 \sigma$, then the input of $T_{s}$ is supra-threshold and the switching process is a practically deterministic one and controlled by $F_{i}(t)$, i.e. $\langle\omega\rangle=\Omega$. It means that the regime of synchronization is realized. The condition $\mu=4 \sigma$ can be considered as the right hand (in respect to maximal noise intensity) synchronization boundary and leads to the following relation for the populations $n_{ \pm}$to observe synchronization:

$$
\Delta n^{m}=n_{ \pm}^{\max }-n_{ \pm}^{\min }>\sqrt{\frac{1}{N+1}}
$$

In other words, synchronization can be observed for any arbitrary small difference between populations by increasing the number of array elements. It means that by using the array we are able to extend the synchronization region in the noise intensity domain up to any arbitrary value.

The presence of common noise $\xi_{c}(t)$ leads to an additional (in respect of $F_{i}(t)$ ) noise-induced correlation of switchings of array elements. Consequently, the last expression and the conclusion should be modified, but, at least, the effect of the enhancement of the synchronization region should still exist. Results of numerical simulations of the triggers array are presented in Fig. 2 for two different combinations of signal amplitude $A$ and noise 

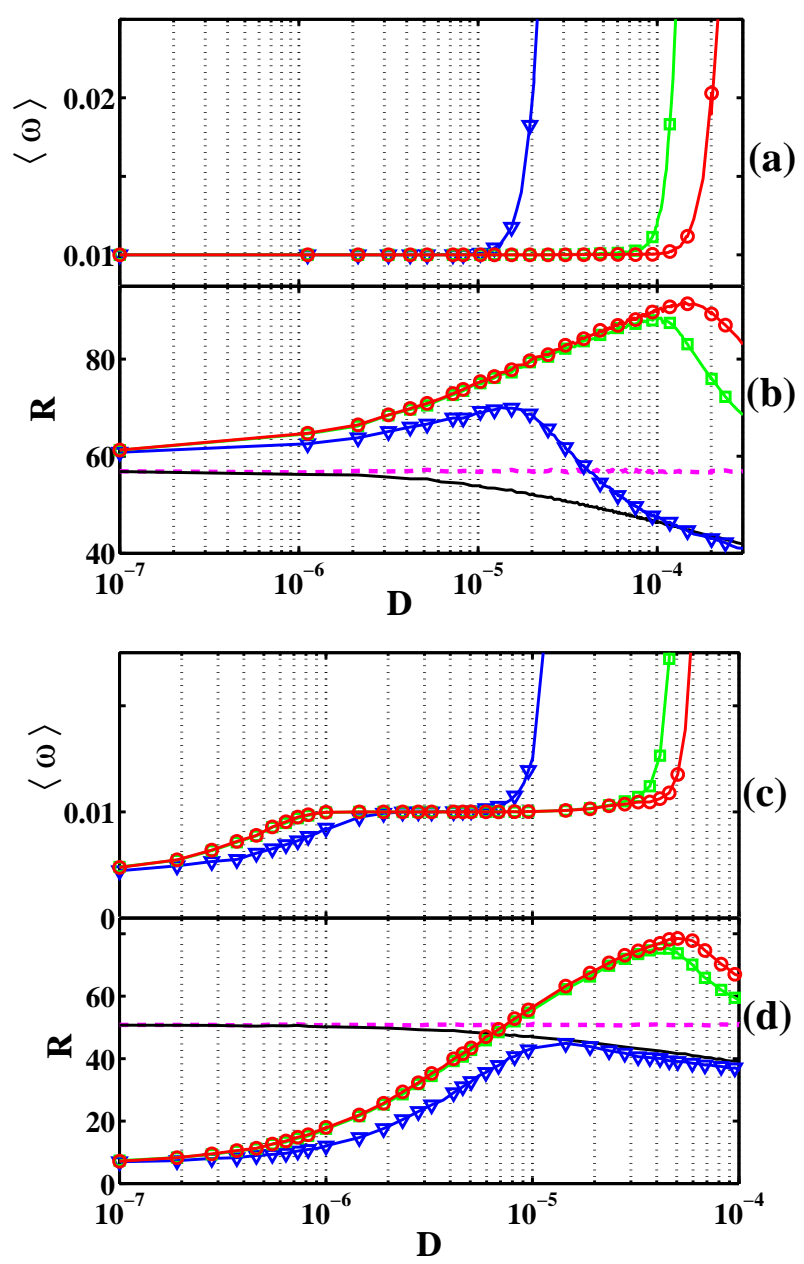

FIG. 2: (Color online) Results of simulations for array of Schmitt triggers. Angular MSR $\langle\omega\rangle$ (a and c) and SNR $R$ (b and d) are shown as functions of internal noise intensity $D$. The parameters are $\Omega=0.01, \tau=1 / 100, U_{0}=1,|\Delta U|=0.1$ for all simulations. Figures (a) and (b) correspond to the parameters values: $A=0.06$ and $D_{c}=0.00001$; figures (c) and (d) correspond to $A=0.025$ and $D_{c}=0.000007$. In figures (a) and (c) markers $\nabla$ (blue lines), $\square$ (green lines) and $\bigcirc$ (red lines) correspond to an array of $N=1, N=50$ and $N=500$ elements respectively. Input SNRs are shown by dashed (magenta) lines (without internal noise, $D=0$ ) and by solid (black) lines (with internal noise, $D \neq 0$ ) in figures (b) and (d). Output SNRs of array are shown by different markers in figures (b) and (d).

intensity $D_{c}$. The extension of the synchronization region is clearly demonstrated by increasing the number of elements. The output SNR, $R$, can exceed both the input SNR of the array [dashed lines in Fig. 2 (b) and (d)], when internal noise is not taken into account, $D=0$, and the input SNR of an individual element [solid lines in Fig. 2 (b) and (d)], when internal noise is taken into consideration, $D \neq 0$. This SNR gain is a non-trivial nonlinear effect, since by tuning internal noise only, one is able to improve the SNR of an external noisy signal. Note that the recently reported SNR gain by Cubero et al [32] has

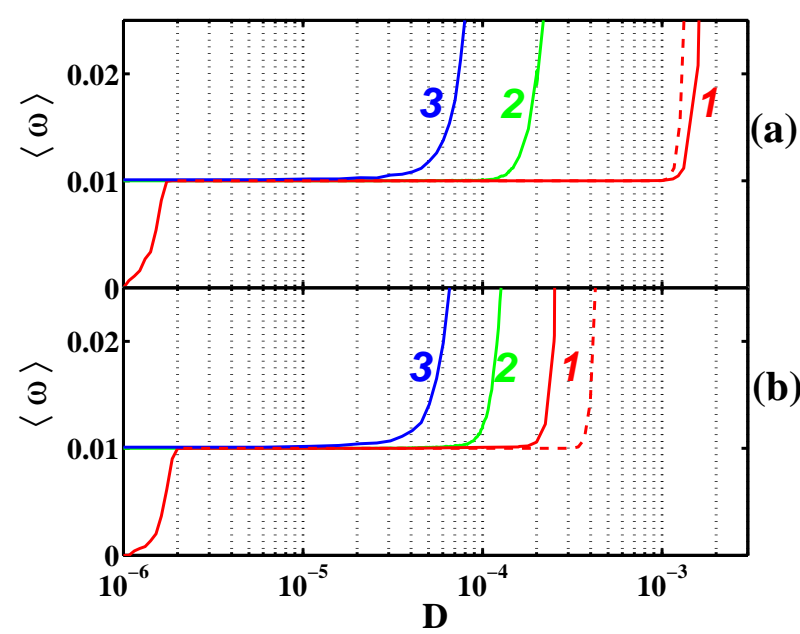

FIG. 3: (Color online) Angular MSRs are shown as functions of internal noise intensity $D$ for different values of common noise intensity: Line 1 (red) $D_{c}=0$, line 2 (green) $D_{c}=0.00001$, line 3 (blue) $D_{c}=0.00002$. The dashed lines correspond to theoretical curves, calculated using expression (9). Figures (a) and (b) correspond to an array with $N=500$ and $N=50$ respectively. Other parameters are specified in the caption of Fig. 2.

been demonstrated when noise in the external signal is absent, i.e. without common noise $D_{c}=0$.

It is clear (Fig. 2) that there is no direct relation between the synchronization and SNR gain, since the gain is observed when there is no synchronization $(\langle\omega\rangle \neq \Omega)$. So it is problematic to use SNR as an indicator of the efficiency of information transmission by spike trains.

The role of common noise is illustrated in Fig.3. The noise leads to a decrease in the synchronization region. Note, that for $D_{c}=0$ the right hand boundary of the synchronization region can be estimated by calculation of the MSR using the following expression:

$$
\langle\omega\rangle=\Omega+2 \pi \gamma / 4\left[1-\Delta r^{2}\left(1-4 \frac{\tanh (\gamma T / 4)}{\gamma T}\right)\right]
$$

where $\gamma=r_{+}(0)+r_{-}(0), \Delta r=\left[r_{-}(0)-r_{+}(0)\right] / \gamma$ and the crossing rates are defined by the expressions:

$$
r_{ \pm}(0)=\frac{1}{2 \pi \tau} \exp \left(-\frac{[ \pm|\Delta U|-\mu(0)]^{2} \tau}{\sigma^{2}(0)}\right)
$$

where $\mu(0)=N\left[2 n_{+}(0)-1\right]$ and $\sigma^{2}(0)=2 N n_{+}(0)[1-$ $\left.n_{+}(0)\right]$, and depend on the number $N$ of array elements. The expression (9) is valid only for $\langle\omega\rangle \geq \Omega$; the first term in (9) reflects the fact that $\mu(0)>\Delta \bar{U}$, whereas the second term is identical to the expression (70) derived in [40]. The correspondence between numerical results and curves defined by (9) becomes visible with increasing $N$, since the error of approximation of the Bernoulli distribution (7) by the continuous normal distribution decreases.

Now let us demonstrate that the effect of synchronization enhancement is observed for arrays with another ba- 


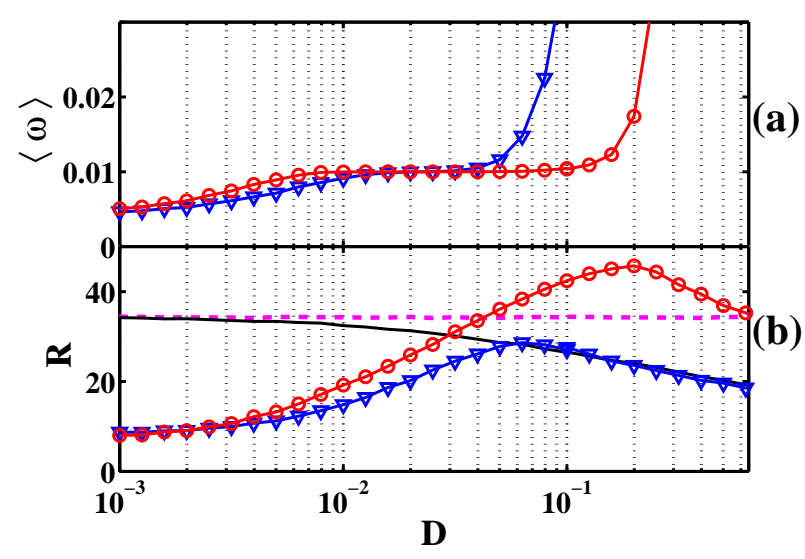

FIG. 4: (Color online) Results of simulations for an array of overdamped bistable oscillators. Angular MSR $\langle\omega\rangle$ (a) and SNR $R$ (b) are shown as functions of internal noise intensity $D$. The parameters are $\Omega=0.01, \tau=1 / 100, A=0.2$ and $D_{c}=0.02$. Markers $\nabla$ (blue lines) and $\bigcirc$ (red lines) correspond to an array of $N=1$ and $N=50$ elements respectively. In figure (b) input SNRs are shown by the dashed (magenta) line (without internal noise, $D=0$ ) and the solid (black) line (with internal noise, $D \neq 0$ ); output SNRs are shown by markers.

sic element. In Fig. 4, the simulation results for bistable overdamped oscillators are shown. In this case, the output $x(t)$ of each element of the array is defined by the following equation:

$$
\dot{x}-x+x^{3}=F(t) .
$$

The summing element was modified in comparison with the trigger case by using the limitation of amplitude of signal which acts on the end element: $S(t)=$ $\tanh \left[\pi \sum_{i}^{N} U^{i}(t)\right], U^{i}=x^{i}(t)$. To model both internal $\xi_{i}(t)$ and common $\xi_{c}(t)$ noise sources, color noise $\xi(t)$ was used with correlation time $\tau=1 / 100$ :

$$
\dot{\xi}+\frac{1}{\tau} \xi=\frac{\sqrt{2 D}}{\tau} \eta(t)
$$

where $\eta(t)$ is white Gaussian noise. Results (Fig.4) demonstrate both synchronization enhancement and SNR gain in the array of bistable oscillators.

\section{EXCITABLE ARRAY}

Finally, we checked the presence of effects in an array of excitable elements that have been modeled by the Fitzhugh Nagumo (FHN) system. The output $U^{i}=u(t)$ of each excitable element obeys the equation:

$$
\begin{array}{r}
\dot{u}=u-\frac{u^{3}}{3}-v \\
\dot{v}=\epsilon[u+a-F(t)],
\end{array}
$$

where $u$ and $v$ represent the membrane potential and slow recovery of a neuron respectively; $\epsilon \ll 1$ and $a$ are

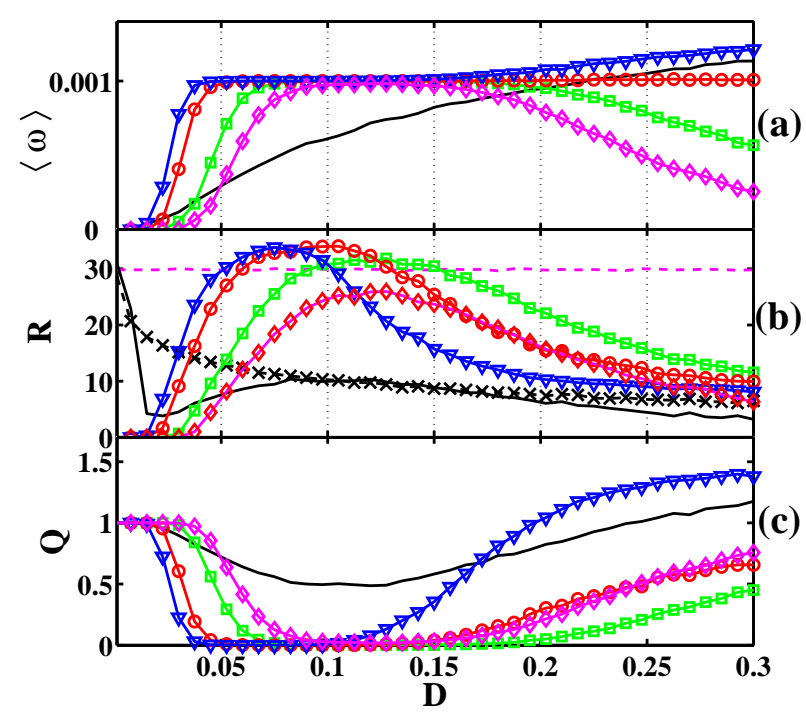

FIG. 5: (Color online) Results of simulations for FHN systems. Angular MSR $\langle\omega\rangle(\mathrm{a})$, SNR $R(\mathrm{~b})$ and the total error $Q$ (c) are shown as functions of internal noise intensity $D$. The parameters are $\Omega=0.001, \tau=1 / 100, \epsilon=0.001, a=1.1$, $A=0.05$ and $D_{c}=0.002, N=50$. The solid (black) lines correspond to the output of one element. Markers correspond to an array $(\mathrm{N}=50)$ with different values of $u_{0}: \diamond$ (magenta lines) $u_{0}=20, \square$ (green lines) $u_{0}=27.5, \bigcirc$ (red lines) $u_{0}=40$, and $\nabla$ (blue lines) $u_{0}=45$. In figure (b) input SNRs are shown by the dashed (magenta) line (without internal noise, $D=0)$ and by markers $\times$ (black line) (with internal noise, $D \neq 0)$.

parameters. Noise sources $\xi_{c}(t)$ and $\xi(t)$ correspond to color noise (12) with $\tau=1 / 100$. The input information signal $F_{i}(t)$ is modified to be close to the output of the FHN system. $F_{i}(t)$ is a sequence of pulses with period $T$, amplitude $A\left(F_{i}(t)\right.$ is varying between 0 and $\left.-A\right)$ and pulse ratio 7 (for an array of triggers and bistable oscillators the pulse ratio is equal to 2). The summing element produces the output signal in the form

$$
S(t)=-0.1\left[\tanh \left(\pi \sum_{i=1}^{N} U^{i}(t)+u_{0}\right)+1\right]
$$

where $U^{i}(t)=u^{i}(t)$. The signal $S(t)$ is limited to the range [0:-0.2] similar to the signal $F_{i}(t)$. The parameter $u_{0}$ defines the zero level of the sum of the output signals. Such a configuration with the summing element $\sum$ having the limitation of an output amplitude and variable mean level reflects the dynamics of the membrane potential of neurons [33].

Since the stationary state of the FHN system (13) corresponds to $u=-a$, then the $u_{0}=-a N / 2$ defines a symmetrical output signal $S(t)$. The signal $S(t)$ acts on the end element $T_{S}$ modeled by the FHN system too. The threshold value of signal amplitude is $A \approx 0.1$ for given $T=2000 \pi$. Results of the simulations for $N=50$ and different values of parameter $u_{0}$ are presented in Fig. 5 . Note that the array of FHN systems differs from the trig- 
gers and bistable oscillators considered above since the FHN system has only one threshold.

In general, the results (Fig. 5) support the conclusion derived from the analysis of triggers and bistable oscillators arrays. However, some differences can be seen. Firstly, the synchronization regime does not occur in one element, whereas an array is able to induce synchronization. Secondly, parameter $u_{0}$ changes significantly the behavior of MSR $\langle\omega\rangle$ and it is difficult to estimate the efficiency of signal processing by using MSR only, because of the presence of only one threshold. Therefore the total error $Q$ for pulse detection [37] was also calculated:

$$
Q=P_{m}+\frac{P_{f}}{n}
$$

where $P_{m}=1-P_{c} / n$ is the fraction of missed pulses, $P_{c}$ is the number of correctly detected pulses and $n$ is the total number of pulses; $P_{f}$ is the number of false positive events, i.e. incorrectly detected pulses. We consider that the pulse is being correctly detected if it follows an input spike. We used the time interval $2 \Delta T$ (here $\Delta T$ is the pulse duration of signal $\left.F_{i}(t)\right)$ after the beginning of the input pulse as the time interval of correct detection.

The total error $Q$ for different values of $u_{0}$ is shown in Fig. 5 (c). The use of an array can provide error-free signal transmission, and parameter $u_{0}$ is an additional one that can be used for transmission optimization. For example, if an array is able to estimate the intensity $D$ of internal noises, then by changing $u_{0}$ the synchronization region can be significantly increased [one can start with $u_{0}=45$ and switch to $u_{0}=27.5$ for $D>0.1$, see Fig. 5 (c)].

\section{CONCLUSIONS}

Summarizing, it has been demonstrated that the nonlinear regime of signal transformation can be optimized for signal processing. Extension of the synchronization region by increasing the size of bistable systems array (triggers and bistable oscillators) has been observed. Array inducing synchronization in the case of excitable systems has been demonstrated. It has been shown theoretically that in the absence of common noise, i.e. a noise-free input signal, that the synchronization region can be arbitrarily extended by increasing the number of bistable elements. The synchronization region decreases with an increase of the intensity of common noise. Also, array induced SNR gain has been demonstrated. For all considered cases the output value of $R$ exceeds its input value in a certain range of internal noise intensity. It has been shown that the presence of the gain does not unambiguously mean an optimal signal transmission. The analysis has revealed some differences between bistable and excitable dynamics and showed evidence that the use of an array leads to a significant decrease of error transmission in comparison with a single system. Let us stress that all results were obtained for an array of uncoupled elements. It is reasonable to expect the extension of the results to arrays with couplings between elements, although differences might also appear.

\section{ACKNOWLEDGMENTS}

The author thankfully acknowledges L. SchimanskyGeier for fruitful, continuing collaboration and encouragement with the most valuable discussions. The author thanks N. Khovanova, A. McCafferty and R. Haley for critical reading of the manuscript, and referees for useful comments and the suggestion to provide details of numerical simulations. The research was supported by the Engineering and Physical Sciences Research Council (UK) and partially by Alexander-von-Humboldt Foundation.

\section{APPENDIX A}

For completeness, here we provide details of numerical simulations of the Langevin equations (2), (11), (12) and (13), and calculations of spectra and MSR $\langle\omega\rangle$.

The equations were presented in the normal form as a set of one-dimensional differential equations:

$$
\dot{x}_{i}=Z_{i}(\mathbf{x})+\sigma_{i} \eta_{i}(t)
$$

where $\mathbf{x}(t)$ is the vector of dynamical variables, $Z_{i}$ represent the deterministic parts of the equations, $\eta_{i}(t)$ is a white noise source with $\langle\eta(t)\rangle=0$ and $\langle\eta(t) \eta(s)\rangle=$ $\delta(t-s)$, and $\sigma_{i}$ is noise amplitude. In the numerical simulations the Heun integration scheme was used as follows [43]:

$$
\begin{gathered}
\tilde{x}_{i}\left(t_{j+1}\right)=x_{i}\left(t_{j}\right)+h Z_{i}\left(\mathbf{x}\left(t_{j}\right)\right)+\sigma_{i} \sqrt{h} G_{i}\left(t_{j}\right) \\
x_{i}\left(t_{j+1}\right)=x_{i}\left(t_{j}\right)+\frac{h}{2}\left[Z_{i}\left(\mathbf{x}\left(t_{j}\right)\right)+Z_{i}\left(\tilde{\mathbf{x}}\left(t_{j+1}\right)\right)\right]+ \\
\sigma_{i} \sqrt{h} G_{i}\left(t_{j}\right),
\end{gathered}
$$

where $h$ is the time step, $t_{j+1}=t_{j}+h$, and $G_{i}\left(t_{j}\right)$ are the Gaussian (normal) random numbers with zero mean and unit dispersion (if $\sigma_{i}$ was equal to zero then the corresponding stochastic term was excluded from the equation). The Box-Muller algorithm [44] was used to generate $G_{i}\left(t_{j}\right)$. An independent random number generator with an independent initialization was used for each noise source $\eta_{i}(t)$. The time step was chosen as $h=T / 2^{n}$, where $T$ is the period of the pulses sequence and $n$ is an integer number. The following values of $n$ were used: $n=17$ for bistable arrays of triggers and overdamped bistable oscillators, and $n=21$ for an array of FHN systems.

The difference scheme used for equation (1) is given by

$$
U\left(t_{j+1}\right)=U_{0} \operatorname{sgn}\left[|\Delta U| \operatorname{sgn}\left[U_{\text {out }}\left(t_{j}\right)\right]-F\left(t_{j}\right)\right] .
$$


The power spectrum $P(\omega)$ was calculated by the periodogram method with a rectangular time window, and signal amplitude spectrum $X(\omega)$ was calculated by the base-2 fast Fourier transform: $P\left(\omega_{j}\right)=$ $\left(\frac{1}{N}\right) \sum_{k=1}^{N} X_{k}^{2}\left(\omega_{j}\right)$, where $N=200$ is the number of periodograms. Each periodogram was calculated from random initial conditions after skipping a relaxation time. The length of the periodogram was equal to $L=65536$ points and the time sampling interval was $\Delta t=2^{m} h$, where $m$ is an integer $(m=6$ for arrays of triggers and bistable overdamped oscillators and $m=10$ for array of FHN systems). These chooses of the sampling interval $\Delta t$ and time step $h=T / 2^{n}$ provide the absence of the leakage effect for a periodic signal of frequency $\Omega=2 \pi / T$, i.e. one frequency bin $\Delta \omega$ contains all the power of signal harmonics $\omega=l \Omega$, where $l=1,2 \ldots$ To avoid aliasing, a low-frequency linear filter with cut-off frequency $\alpha=\pi /(2 \Delta t)$ was used of the form:

$$
\dot{x_{f}}=-\alpha x_{f}+\alpha x \text {. }
$$

The filter output $x_{f}(t)$ was used for the spectrum calculation, whereas $x(t)$ corresponds to the considered signals. These can be the output of an array unit $U^{i}(t)$, the output of array $U_{\text {out }}(t)$, or the input signal $F_{i}(t)+\xi_{c}(t)+\xi_{i}(t)$ with or $F_{i}(t)+\xi_{c}(t)$ without internal noise.

As follows from $[38,39]$, the output signals $U^{i}(t)$ and $U_{\text {out }}(t)$ consist of periodic and noisy components. The periodic component corresponds to the informational signal $F_{i}(t)$ and contains discrete constituents $P(k \Omega)=$ $S_{k} \delta(\omega-k \Omega)$, where $k$ is an integer number, i.e. it contains harmonics of frequency $\Omega$. The spectrum calculation methos described above defines the weight of $\delta$-peak on the frequency $\Omega$ as $S_{i}(\Omega)=P(\Omega)$.

Following $[1,2,30]$ the noise component $S_{n}(\Omega)$ was estimated using the power spectrum $P(\Omega)$ in the following way

$$
S_{n}(\Omega)=\Delta \omega \frac{1}{2 m}\left[\sum_{j=i-l, j \neq i}^{j=i+l} P\left(\omega_{j}\right)\right]
$$

where $\Delta \omega=2 \pi /(L \Delta t)$ is the frequency resolution in the numerically calculated power spectrum $P\left(\omega_{j}\right) ; \omega_{i}=\Omega$ and $l=6$ defines a bandwidth to approximate the noise background at signal frequency $\Omega$.

Note that the input $R$ allows verification of the results of numerical simulations, since the input $R$ for a periodic sequence of rectangular pulses $F_{i}(t)$ can be calculated directly as

$$
R=10 \log _{10} \frac{A^{2}}{2\left(D_{c}+D\right) \Delta \omega} .
$$

For the parameters given above the error of SNR calculations is less than $1 \mathrm{~dB}$, i.e. it is comparable with size of the markers in the figures.

For the calculation of $\operatorname{MSR}\langle\omega\rangle$, time intervals $\Delta t_{j}=$ $t_{j}-t_{j-1}$ between two successive transitions from the given state to another state (for bistable arrays) and between two successive spikes (for an excitable array) were determined in parallel to periodogram calculations. To determine the transition moment $t_{j}$ the output $x(t)$ of the bistable oscillator (11) was additionally filtered by a symmetrical trigger with thresholds $\Delta U= \pm 0.5$ to produce a dichotomous (two-state) signal $x_{d}(t)$ confined to the values $\pm\left|x_{m}\right|$ only, where $x_{m}=1$. Similar filtering was used for the output $u(t)$ of the FHN system. MSR was determined as $\langle\omega\rangle=2 \pi /\langle\Delta t\rangle$, where $\langle\Delta t\rangle=\left(\frac{1}{M}\right) \sum_{j=1}^{M} \Delta t_{j}$ and $M$ is the number of transitions (spikes) during the simulation time. The simulation time was always larger than 5000 periods $T$ of the information component $F_{i}(t)$.
[1] L. Gammaitoni, P. Hänggi, P. Jung, and F. Marchesoni, Rev. Mod. Phys. 70, 223 (1998).

[2] V. S. Anishchenko, A. B. Neiman, F. Moss, and L. Schimansky-Geier, Phys. Usp. 42, 7 (1999).

[3] R. Benzi, A. Sutera, and A. Vulpiani, J. Phys. A: Math. Gen. 14, L453 (1981).

[4] C. Nicolis, Tellus 34, 1 (1982).

[5] A. Destexhe, and D. Contreras, Science 314, 85 (2006).

[6] J. J. Collins, C. C. Chow, A. C. Capela, and T. T. Imhoff, Phys. Rev. E 54, 5575 (1996).

[7] J. F. Lindner, B. K. Meadows, W. L. Ditto, M. E. Inchiosa, and A. R. Bulsara, Phys. Rev. Lett. 75, 3 (1995).

[8] M. E. Inchiosa, and A. R. Bulsara, Phys. Rev. E 53, R2021 (1996).

[9] U. Siewert, and L. Schimansky-Geier, Phys. Rev. E 58, 2843 (1998).
[10] J. J. Collins, C. C. Chow, and T. T. Imhoff, Nature (London) 376, 236 (1995).

[11] P. C. Gailey, A. Neiman, J. J. Collins, and F. Moss, Phys. Rev. Lett. 79, 4701 (1997).

[12] D. R. Chialvo, A. Longtin, and J. Müller-Gerking, Phys. Rev. E 55, 1798 (1997).

[13] N. G. Stocks, Phys. Rev. Lett. 84, 2310 (2000); N. G. Stocks, and R. Mannella, Phys. Rev. E 64, 030902(R) (2001); D. Rousseau, F. Duan, and F. ChapeauBlondeau, Phys. Rev. E 68, 031107 (2003); A. Nikitin, N. G. Stocks, and R. P. Morse, Phys. Rev. E 75, 021121 (2007).

[14] A.-H. Sato, M. Ueda, and T. Munakata, Phys. Rev. E 70, 021106 (2004)

[15] A. Neiman, L. Schimansky-Geier, and F. Moss, Phys. Rev. E 56, R9 (1997). 
[16] F. Rieke, D. Warland, R. de Ruyter van Steveninck, and W. Bialek, Spikes: Exploring the Neural Code (Cambridge, Massachusetts: The MIT Press; 1999).

[17] R. Bartussek, P.Hänggi, and P. Jung, Phys. Rev. E 49, 3930 (1994).

[18] A. Neiman, in Stochastic Processes in Physics, Chemistry, and Biology, Edited by J.A. Freund and T. Poeschel (Springer, Berlin, 2000) p. 190.

[19] B. Shulgin, A. Neiman, and V. Anishchenko, Phys. Rev. Lett. 75, 4157 (1995)

[20] K. Loerincz, Z. Gingl, and L. B. Kiss, Phys. Lett. A 224, 63 (1996)

[21] I. A. Khovanov, and V. S. Anischenko, in Applied Nonlinear Dynamics and Stochastic Systems Near the Millennium, edited by J.B. Kadtke and A. Bulsara, AIP Conf. Proc. 411 (AIP, Woodbury, New York, 1997) p. 267.

[22] J. A. Freund, A. B. Neiman, and L. Schimansky-Geier, Europhys. Lett. 50, 8 (2000).

[23] R. Rozenfeld, J. A. Freund, A. B. Neiman, and L. Schimansky-Geier, Phys. Rev. E 64, 051107 (2001).

[24] J. Casado-Pascual et al, Phys. Rev. E 71, 011101 (2005).

[25] I. A. Khovanov, and P. V. E. McClintock, Phys. Rev. E 76, 031122 (2007).

[26] A. Neiman, L. Schimansky-Geier, F. Moss, B. Shulgin, and J. J. Collins, Phys. Rev. E 60, 284 (1999).

[27] S. Bahar, A. Neiman, L. A. Wilkens, and F. Moss, Phys. Rev. E 65, 050901(R) (2002).

[28] V. Anishchenko, A. Neiman, A. Astakhov, T. Vadivasova, and L. Schimansky-Geier, Chaotic and Stochastic Processes in Dynamical Systems, Springer-Series on Synergetics (Springer, Berlin, 2002).

[29] Z. Gingl, P. Makra, and R. Vajtai, Fluct. Noise Lett. 1, L181 (2001).
[30] J. Casado-Pascual, J. Gómez-Ordóñez, M. Morillo, and P. Hänggi, Phys. Rev Lett. 91, 210601 (2003).

[31] I. A. Khovanov, and P. V. E. McClintock, Phys. Rev. E 67, 043901 (2003).

[32] D. Cubero, J. Casado-Pascual, J. Gómez-Ordóñez, J. M. Casado, and M. Morillo, Phys. Rev. E 75, 062102 (2007).

[33] P. Poirazi, T. Brannon, and B. W. Mel, Neuron 37, 989 (2003).

[34] D. Durstewitz, and J. K. Seaman, Neuroscience 139, 119 (2006); A. A. Koulakov, S. Raghavachari, A. Kepecs, and J. E. Lisman, Nat. Neurosc. 5, 775 (2002).

[35] P. Jung, Phys. Rev E 50, 2513 (1994); P. Jung, A. Neiman, M. K. N. Afghan, S. Nadkarni, and G. Ullah, New J. Phys. 7, 17 (2005).

[36] H. van Trees, Detection, Estimation and Modulation Theory (Wiley, New York, 1978).

[37] G. Wenning, T. Hoch, and K. Obermayer, Phys. Rev. E 71, 021902 (2005).

[38] R. L. Stratonovich, Topics in theory of random noise (New-York: Gordon and Breach, 1963).

[39] P. Jung, Phys. Rep. 234, 175 (1993).

[40] J. Casado-Pascual et al, Phys. Rev. E 71, 011101 (2005).

[41] B. McNamara, and K. Wiesenfeld, Phys. Rev. A 39, 4854 (1989).

[42] W. Feller, An Introduction to Probability Theory and its Applications (New York: John Wiley \& Sons, 1968).

[43] P. E. Kloeden, and E. Platen, Numerical solution of stochastic differential equations (Springer-Verlag, Berlin, 1992).

[44] W. H. Press, B. P. Flannery, S. A. Teukolsky, and W. T. Vetterling, Numerical recipes: the Art of Scientific Computing (CUP, Cambridge, 1986). 